\title{
The Logic of Exact Covers: Completeness and Uniform Interpolation
}

\author{
Dirk Pattinson \\ Research School of Computer Science, The Australian National University \\ Department of Computing, Imperial College London
}

\begin{abstract}
We show that all (not necessarily normal or monotone) modal logics that can be axiomatised in rank-1 have the interpolation property, and that in fact interpolation is uniform if the logics just have finitely many modal operators. As immediate applications, we obtain previously unknown interpolation theorems for a range of modal logics, containing probabilistic and graded modal logic, alternating temporal logic and some variants of conditional logic.

Technically, this is achieved by translating to and from a new (coalgebraic) logic introduced in this paper, the logic of exact covers. It is interpreted over coalgebras for an endofunctor on the category of sets that also directly determines the syntax. Apart from closure under bisimulation quantifiers (and hence interpolation), we also provide a complete tableaux calculus and establish both the Hennessy-Milner and the small model property for this logic.
\end{abstract}

\section{INTRODUCTION}

This paper can be seen from two different angles. From the perspective of universal coalgebra [35], we introduce and investigate a new coalgebraic logic, the logic of exact covers, interpreted over coalgebras for an endofunctor that also directly induces the syntax. As a contribution to modal logic, we prove previously unknown interpolation theorems for a range of modal logics, more precisely, for all modal logics that can be axiomatised in rank-1.

Both views are linked as all rank-1 logics can be equipped with coalgebraic semantics (see [38] for a detailed discussion): for every rank-1 logic $L$ there is a functor $T_{L}$ so that $L$ is sound and complete over $T_{L}$-coalgebras. While this may not be the natural semantics for the logic under consideration, it nonetheless allows us to apply coalgebraic techniques when working with the logic. In particular, we can translate back and forth between $L$ and the logic of exact covers induced by $T_{L}$. This translation allows us to transfer the interpolation theorem that we establish for the logic of exact covers, back to the logic $L$.

Our results apply to all rank- 1 logics, i.e. logics that can be axiomatised by formulae whose modal nesting depth is uniformly equal to one. While this excludes logics like S4 and $\mathrm{T}$, it covers a large variety of non-normal (and sometimes non-monotone) modal logics such as graded and probabilistic modal logic, alternating temporal logic and various flavours of conditional logics for which we believe the interpolation theorems are new.

The main technical vehicle used in this paper, the logic of exact covers, can be seen as a logic in the style of Moss' coalgebraic logic [26], an extension of classical propositional calculus with the so-called cover modality. It is interpreted over coalgebras for a set-endofunctor $T$ that directly determines the syntax. Here, we think of coalgebras $(C, \gamma: C \rightarrow T C)$ as frames in the sense of modal logic where the endofunctor $T$ determines the structure of the frame: different choices of $T$ induce, e.g., Kripke frames, monotone neighbourhood frames or probabilistic frames. Coalgebraic logic is by now welldeveloped: Venema presents automata for fixpoint logics based on the cover modality [40], complete axiomatisations may be found in [4], [18] and Moss' original paper [26] establishes the Hennessy-Milner property.

While coalgebraic logic provides a generic treatment of many different classes of frames, all of the above results rely on the assumption that the functor that defines the frame classes preserves weak pullbacks which precludes the instantiation of the generic theory to important examples such as (monotone) neighbourhood frames [15] or conditional frames [8]. The conceptual reason for requiring that the underlying endofunctor preserve weak pullbacks lies at the very heart of the definition of the semantics of the cover modality: the endofunctor needs to be lifted to relations and weak pullback preservation is required to ensure functoriality of this lifting.

The question of possible ways to extend the treatment of the cover modality to classes of (coalgebraic) frames without requiring the preservation of weak pullbacks has so far remained largely open and only partial answers can be found in the literature. Taking formulas to be elements of the final sequence of the underlying endofunctor removes the requirement for weak pullback preservation [27], but does not provide a syntactical notion of modal operator.

For the case of monotone neighbourhood frames (where weak pullback preservation fails), it has been demonstrated that the cover modality is definable by changing the notion of relation lifting [36]. This was later generalised by Marti and Venema [25] for functors that have a lax extension preserving diagonals, but Theorem 2 of op.cit. acknowledges that this must necessarily fail for (not necessarily monotone) neighbourhood frames.

Here, we take a different approach. Given that the extension of an arbitrary endofunctor $T:$ Set $\rightarrow$ Set to relations is problematic, we argue that the semantics of modal formulas should not be defined by extending $T$ to relations, but simply by applying $T$ to functions, where logical properties can be derived in terms of the functor laws. Rather than defining the semantics of the cover modality by applying (the lifting of) $T$ to the satisfaction relation we propose an alternative semantics 
that arises by applying $T$ to the theory map. In particular, this entails that logics conceived in this way are no longer monotonic which precludes e.g. fixpoint extensions in the style of [40]. Our main findings indicate that this is nonetheless a viable approach that leads to a rich theory: we establish the Hennessy-Milner property and the small model property, provide a complete tableau calculus and show that the logic of exact covers is closed under bisimulation quantifiers (and hence admits uniform interpolation).

To establish the link between modal logics in the standard sense (that we take to be sets of formulae closed under congruence, modus ponens, propositional tautologies and uniform substitution) we equip the latter with coalgebraic semantics as in [38]: assuming $L$ is a rank-1 logic, we obtain an endofunctor $T_{L}$ so that $L$ is sound and complete for $T_{L}$-coalgebras. In particular, both the logic $L$ and the logic of exact covers induced by $T_{L}$ can be interpreted over $T_{L}$-coalgebras. To transfer interpolation from the logic of exact covers to the given rank-1 $\operatorname{logic} L$ we provide a bi-directional semanticspreserving translation between $L$ and the associated logic of exact covers and interpolation for $L$ follows from the corresponding result for the logic of exact covers by soundness and completeness. Concrete instances of our results establish uniform interpolation for the classical and monotone modal logic [8], alternating temporal logic [3] and the expressively equivalent coalition logic [33], the basic conditional logic $C K$ as well as extensions of $C K$ with rank-1 axioms.

Related Work. The logic of exact covers can be seen as a non-monotonic version of Moss' coalgebraic logic [26] that overcomes the requirement of weak-pullback preservation and is thus applicable to a much larger class of (coalgebraic) frames. The proof strategy for establishing the HennessyMilner property for the logic of exact covers follows [37], and the interpolation proof is similar to [36] but at a higher level of generality for a structurally different (non-monotonic) interpretation of the logic. Uniform interpolation via bisimulation quantifiers (as well as many other methods) is discussed in [9]. Uniform interpolation for the modal logic $K$ has been established in [42], [12] and [36] establishes uniform interpolation for monotone modal logic.

Organisation of the Paper. The reader is invited to skip (but encouraged to consult) Section II where we mainly settle notation. The logic of exact covers is introduced in Section III where we also illustrate the formalism with two concrete examples. Readers with an interest in coalgebraic semantics and/or logics may wish to continue with Sections IV, V and VI where we establish the Hennessy-Milner property, the small model property and a complete tableaux calculus, respectively, using mostly standard methods. Those mainly interested in interpolation may wish to skip those sections and jump to Section VII (coming back to the earlier sections as necessary) where we establish closure under bisimulation quantifiers which is subsequently used in Section VIII to obtain the interpolation results for rank-1 logics. We conclude with a discussion of our results and one open problem in Section IX.

\section{PRELIMINARIES AND Notation}

Sets and Functions. If $X$ is a set, we write $|X|$ for its cardinality. For functions $f: X \rightarrow Y, f^{-1}(B)=\{x \in X \mid$ $f(x) \in B\}$ denotes the inverse image of $B \subseteq Y$ under $f$ and $f[A]=\{f(a) \mid a \in A\}$ the direct image of $A \subseteq X$. Note that $f\left[f^{-1}(B)\right] \subseteq B$ whereas $A \subseteq f^{-1}(f[A])$ for all $A \subseteq X$ and $B \subseteq Y$. If $X$ is a set, then $\operatorname{ch}(X)$ denotes the set of choice functions on $X$, that is, the set of functions $f: X \rightarrow \bigcup X$ such that $f(x) \in x$ for all $x \in X$.

Modal Logic. We assume familiarity with the basic concepts of modal logic, found e.g. in [6]. A modal similarity type is a set $\Lambda$ of modal operators with associated arities. We fix a countable set $\mathcal{V}$ of propositional variables throughout. If $\mathcal{V}_{0} \subseteq \mathcal{V}$ is a set of propositional variables, the set of modal formulae induced by $\mathcal{V}_{0}$ and $\Lambda$ is given by the grammar $\mathcal{F}_{\mathcal{V}_{0}} \ni \phi, \psi::=\perp|\phi \wedge \psi| \phi \vee \psi|\neg \phi| \varnothing\left(\phi_{1}, \ldots, \phi_{n}\right)$ where $p \in \mathcal{V}_{0}$ and $\varnothing \in \Lambda$ is $n$-ary. We abbreviate $\mathcal{F}_{\mathcal{V}}(\Lambda)$ to $\mathcal{F}(\Lambda)$. If $F$ is a set (of formulae) we write $\operatorname{Prop}(F)$ for the set of propositional formulae over $F$ and $\Lambda(F)=$ $\left\{\varnothing\left(\phi_{1}, \ldots, \phi_{n}\right) \mid \phi_{1}, \ldots, \phi_{n} \in F\right.$ and $\varnothing \in \Lambda$ is $n$ ary $\}$. The formulae of basic modal logic are given by the similarity type $\Lambda=\{\square\}$ with $\square$ unary and we write $\mathcal{F}(\square)$ instead of $\mathcal{F}(\{\square\})$. A $\Lambda$-logic is a set $L \subseteq \mathcal{F}(\Lambda)$ that contains all propositional tautologies, is closed under uniform substitution and the congruence rules: from $\phi_{i} \leftrightarrow \psi_{i}$ for $i=1, \ldots, n$ infer that $\varnothing\left(\phi_{1}, \ldots, \phi_{n}\right) \leftrightarrow \nabla\left(\psi_{1}, \ldots, \psi_{n}\right)$ for every $n$-ary $\varnothing \in \Lambda$. We write $L \vdash \phi$ if $\phi \in L$. A $L$-unifier of a formula $\phi \in \mathcal{F}(\Lambda)$ is a substitution $\sigma: \mathcal{V} \rightarrow \mathcal{F}(\Lambda)$ such that $\phi \sigma \in L$ and a subset $\Phi \subseteq \mathcal{F}(\Lambda)$ is $L$-consistent if $L \forall \wedge \Psi \rightarrow \perp$ for all finite $\Psi \subseteq \Phi$. A set $\Phi$ of formulae is satisfiable in a given model class if there exists a model / world pair $\mathbb{C}, c$ in this class such that all $\phi \in \Phi$ are true at world $c$ in model $\mathbb{C}$. We write PL for the set of propositional tautologies (that incidentally qualifies as a $\emptyset$-logic).

Kripke models and neighbourhood models. A Kripke model is a triple $\mathbb{M}=(W, R, \theta)$ where $W$ is a set (of worlds), $R \subseteq$ $W \times W$ is an (accessibility) relation and $\theta: V \rightarrow \mathcal{P}(W)$ is a valuation (of the propositional variables). We adopt the standard semantics where $\mathbb{M}, w \models \square \phi$ if $w^{\prime} \models \phi$ for all $w^{\prime}$ with $w R w^{\prime}$ and $\mathbb{M}, w=\diamond \phi$ if there is some $w^{\prime}$ with $w R w^{\prime}$ and $\mathbb{M}, w^{\prime}=\phi$. A monotone neighbourhood model is a triple $(W, \eta, \theta)$ where $W$ is a set (of worlds), $\eta: W \rightarrow \mathcal{P} \mathcal{P}(W)$ is a (neighbourhood) function satisfying $N^{\prime} \in \eta(w)$ whenever $N \subseteq N^{\prime} \subseteq W$ and $N \in \eta(w)$ and $\theta$ is a valuation as above. We adopt the standard semantics where $\mathbb{M}, w \models \square \phi$ iff there exists $N \in \eta(w)$ with $N \subseteq\left\{w^{\prime} \in W \mid \mathbb{M}, w^{\prime}=\phi\right\}$ and $\mathbb{M}, w \models \Delta \phi$ iff $N \cap\left\{w^{\prime} \in W \mid \mathbb{M}, w^{\prime}=\phi\right\} \neq \emptyset$ for all $N \in \eta(w)$.

Coalgebras and Models. Throughout, $T$ : Set $\rightarrow$ Set denotes an endofunctor on the category Set of sets and functions. A $T$-coalgebra is a pair $(C, \gamma)$ where $C \in$ Set is a set (of possible worlds) and $\gamma: C \rightarrow T C$ a (transition) function. A $T$-model is a triple $(C, \gamma, \pi)$ where $(C, \gamma)$ is a $T$-coalgebra and $\pi: C \rightarrow \mathcal{P}(\mathcal{V})$ is a valuation where $\mathcal{V}$ is a countable set of propositional variables as above. For a 
subset $\mathcal{V}_{0} \subseteq \mathcal{V}$ of propositional variables, a $\mathcal{V}_{0}$-morphism $f$ : $(C, \gamma, \pi) \rightarrow(D, \delta, \tau)$ of $T$-models is a function $f: C \rightarrow D$ that satisfies $\delta \circ f=T f \circ \gamma$ and $\mathcal{V}_{0} \cap(\tau \circ f(c))=\mathcal{V}_{0} \cap \pi(c)$ for all $c \in C$. We simply speak of a morphism of $T$-models if $\mathcal{V}_{0}=\mathcal{V}$. We denote the category of $T$-models and $\mathcal{V}$ morphisms by $\operatorname{Mod}(T)$.

Inclusion-Preserving Functors. We write $i_{X \hookrightarrow Y}$ for the inclusion function if $Y$ is a set and $X \subseteq Y$. A functor $T:$ Set $\rightarrow$ Set preserves inclusions if $T i_{X \hookrightarrow Y}=i_{T X \hookrightarrow T Y}$. It is known ([1], see also [2, Theorem III.4.5]) that every functor $T$ can be modified to an inclusion-preserving functor $T^{\prime}$ so that $T$ and $T^{\prime}$ are naturally isomorphic when restricted to the category of nonempty sets (in particular this implies that $\operatorname{Mod}(T)$ and $\operatorname{Mod}\left(T^{\prime}\right)$ are isomorphic). We therefore assume without loss of generality that $T$ preserves inclusions whenever convenient. An inclusion-preserving functor $T$ is called finitary if $T X=\bigcup\{T Y \mid Y \subseteq X$ finite $\}$.

Categorical Notions. We write BA for the category of boolean algebras, $U: \mathrm{BA} \rightarrow$ Set for the forgetful functor and $F$ for its left adjoint (the free construction). The contravariant powerset functor is denoted by $\mathcal{L}$ : Set $\rightarrow \mathrm{BA}^{\mathrm{op}}$ and Uf $: \mathrm{BA}^{\mathrm{op}} \rightarrow$ Set is its right adjoint (the ultrafilter functor). The contravariant powerset functor conceived as an operation on sets is written $\mathcal{Q}: \operatorname{Set}^{\text {op }} \rightarrow$ Set ( so that $\mathcal{Q}=U \circ \mathbb{2}^{\mathrm{op}}$ ).

\section{SyntaX, SEMANTICS AND EXAMPLES}

For the whole section, we fix an endofunctor $T:$ Set $\rightarrow$ Set and begin by introducing syntax and semantics of the logic of exact covers induced by $T$.

Definition 1 (Formulae and Interpretation). The set of formulae of the logic of exact covers over $T$ and a subset $\mathcal{V}_{0} \subseteq \mathcal{V}$ is given by the grammar

$$
\mathcal{F}_{\mathcal{V}_{0}}(T) \ni \phi, \psi::=\top|p| \phi \wedge \psi|\phi \vee \psi| \neg \phi \mid \nabla_{\Sigma}(\mathcal{X})
$$

where $p \in \mathcal{V}_{0}$ and $\mathcal{X} \in T \mathcal{P}(\Sigma)$ and $\Sigma \subseteq \mathcal{F}_{\mathcal{V}_{0}}(T)$ is finite. We write $\mathcal{F}(T)$ for $\mathcal{F}_{\mathcal{V}}(T)$. If $\mathbb{C}=(C, \gamma, \pi) \in \operatorname{Mod}(T)$ is a $T$-model, we define satisfaction $\mathbb{C}, c \models \phi$ in the usual way for the propositional connectives, together with the clauses

$$
\begin{aligned}
\mathbb{C}, c \models p & \Longleftrightarrow p \in \pi(c) \\
\mathbb{C}, c \models \nabla_{\Sigma}(\mathcal{X}) & \Longleftrightarrow T t_{\Sigma}^{\mathbb{C}} \circ \gamma(c)=\mathcal{X}
\end{aligned}
$$

with $t_{\Sigma}^{\mathbb{C}}: C \rightarrow \mathcal{P}(\Sigma)$ the local theory map given by $t_{\Sigma}^{\mathbb{C}}(c)=$ $\{\phi \in \Sigma|\mathbb{C}, c|=\phi\}$.

Remark 2 (Cover Modality). Our formulae are similar to those of coalgebraic logic [26] where the $\nabla$-modality is (also) defined in terms of functor application. In op.cit., we have modal formulae $\nabla \Phi$ where $\Phi \in T \Sigma$ for a set of formulae $\Sigma$ and the semantics is given in terms of relation lifting. Here, we construct modal formula $\nabla_{\Sigma} \Phi$ from $\Phi \in T \mathcal{P}(\Sigma)$ for a set $\Sigma$ of formulae which allows us to think of the argument of $T$ as a set of (local) theories which is also reflected in the semantics.

We illustrate the semantics of the language just introduced in terms of two extended examples, Kripke models (that also fall within the framework of the cover modality) and monotone neighbourhood models (that fall outside the scope of the cover modality). While Kripke models illustrate the logic of exact covers in a standard, well-understood context, we have chosen monotone neighbourhood models to enable comparison with [36] where uniform interpolation for monotone neighbourhood models is established along similar lines.

Kripke Models. For the entire subsection we assume that $T=\mathcal{P}$, the covariant powerset functor. It is standard (and easy to see) that $\mathcal{P}$-models are in $1-1$ correspondence with Kripke models. For $T=\mathcal{P}$, modal formulae take the shape $\nabla_{\Sigma}\left\{\Phi_{1}, \ldots, \Phi_{n}\right\}$ where $\Phi_{1}, \ldots, \Phi_{n} \subseteq \Sigma$. If $\mathbb{C}=(C, \gamma, \pi)$ is a $T$-model, we have that

$$
\begin{aligned}
\mathbb{C}, c \mid \nabla_{\Sigma}\left\{\Phi_{1}, \ldots,\right. & \left.\Phi_{n}\right\} \Longleftrightarrow \\
& \left\{t_{\Sigma}^{\mathbb{C}}\left(c^{\prime}\right) \mid c^{\prime} \in \gamma(c)\right\}=\left\{\Phi_{1}, \ldots, \Phi_{n}\right\}
\end{aligned}
$$

i.e., the local theories of all successors $c^{\prime} \in \gamma(c)$ are precisely $\Phi_{1}, \ldots, \Phi_{n}$. This amounts to a bisimulation-like condition

- for all $c^{\prime} \in \gamma(c)$ there is $\Phi_{i} \in \Phi$ such that $t\left(c^{\prime}\right)=\Phi$

- for all $\Phi_{i} \in \Phi$ there is $c^{\prime} \in \gamma(c)$ such that $t\left(c^{\prime}\right)=\Phi$ where $t=t_{\Sigma}^{\mathbb{C}}$ is the local theory map. If $\Sigma=\{\phi\}$ this gives the semantic equivalences

$$
\square \phi \equiv \nabla_{\Sigma} \emptyset \vee \nabla_{\Sigma}\{\{\phi\}\} \text { and } \diamond \phi \equiv \nabla_{\Sigma}\{\emptyset,\{\phi\}\} \vee \nabla_{\Sigma}\{\{\phi\}\}
$$

that allow us to translate the standard modal language into $\mathcal{F}(\mathcal{P})$. Note that the left disjunct in the left hand clause expresses the fact that a world has no successors and the right hand disjunct is valid only if a world has at least one successor, and all successors satisfy $\phi$. Dually, we can express $\mathcal{F}(T)$ in the language of $\square$ and $\diamond$ via

$$
\begin{aligned}
& \nabla_{\Sigma}\left\{\Phi_{1}, \ldots, \Phi_{n}\right\} \equiv \\
& \square \bigvee_{1 \leq i \leq n}\left(\bigwedge \Phi_{i} \wedge \neg \bigvee\left(\Sigma \backslash \Phi_{i}\right)\right) \wedge \bigwedge_{1 \leq i \leq n} \diamond\left(\bigwedge \Phi_{i}\right) \wedge \neg \bigvee\left(\Sigma \backslash \Phi_{i}\right)
\end{aligned}
$$

where we note that $\nabla$ also expresses negative information. As a consequence, the logic of exact covers and the modal logic $\mathrm{K}$ are equi-expressive.

Proposition 3 (Translatability for Kripke models). The languages $\mathcal{M L}$ and $\mathcal{F}(\mathcal{P})$ can be effectively translated into one another. More precisely, there are computable functions $s: \mathcal{M L} \rightarrow \mathcal{F}(\mathcal{P})$ and $t: \mathcal{F}(\mathcal{P}) \rightarrow \mathcal{M L}$ such that

$\mathbb{C}, c \models \phi \Longleftrightarrow \mathbb{C}, c=s(\phi)$ and $\mathbb{C}, c \models \psi \Longleftrightarrow \mathbb{C}, c \mid=t(\psi)$

for all $\mathcal{P}$-models $\mathbb{C}=(C, \gamma, \pi)$, all $c \in C$ and all formulae $\phi \in \mathcal{F}(\mathcal{P})$ and $\psi \in \mathcal{M L}$.

Monotone Neighbourhood Models. While the covariant powerset functor $\mathcal{P}$ preserves weak pullbacks (and is therefore within the scope of coalgebraic logic [26]) this is not the case for the functor $\mathcal{M}$ that is given by

$$
\mathcal{M}(X)=\{S \subseteq \mathcal{P} \mathcal{P}(X) \mid S \text { upwards closed }\}
$$

that induces monotone neighbourhood frames [8] as $\mathcal{M}$ models. It has been demonstrated in [36] that the notion of 
relation lifting used to define coalgebraic logic can be modified to accommodate $\mathcal{M}$-models. However this modification is specific to $\mathcal{M}$ and not generic. As the logic of exact covers does not require weak pullback preservation, monotone neighbourhood models are well within its scope.

For $T=\mathcal{M}$, modal formulae take the shape $\nabla_{\Sigma}\left\{\alpha_{1}, \ldots, \alpha_{n}\right\}$ where $\alpha_{1}, \ldots, \alpha_{n} \subseteq \mathcal{P}(\Sigma)$, i.e. each $\alpha_{i}$ is a set of local theories, and $\left\{\alpha_{1}, \ldots, \alpha_{n}\right\}$ is upwards closed. As for the case of Kripke models, satisfaction is characterised by a bisimulation-like condition. We have that $\mathbb{C}, c=\nabla_{\Sigma}(\mathcal{X})$ if

- for all $N \in \gamma(c)$ there is $\alpha \in \mathcal{X}$ such that $\alpha \subseteq t[N]$

- for all $\alpha \in \mathcal{X}$ there is $N \in \gamma(c)$ such that $N \subseteq t^{-1}(\alpha)$ where again $t=t_{\Sigma}^{\mathbb{C}}: C \rightarrow \mathcal{P}(\Sigma)$ is the local theory map. Based on this characterisation, we obtain the equivalences

$$
\begin{aligned}
\square \phi & \equiv \nabla_{\Sigma}\left\{\alpha_{0}, \alpha_{1}, \alpha_{2}, \alpha_{3}\right\} \vee \nabla_{\Sigma}\left\{\alpha_{1}, \alpha_{2}, \alpha_{3}\right\} \vee \nabla_{\Sigma}\left\{\alpha_{2}, \alpha_{3}\right\} \\
\diamond \phi & \equiv \nabla_{\Sigma} \emptyset \vee \nabla_{\Sigma}\left\{\alpha_{2}, \alpha_{3}\right\} \vee \nabla_{\Sigma}\left\{\alpha_{3}\right\}
\end{aligned}
$$

where $\Sigma=\{\phi\}, \alpha_{0}=\emptyset, \alpha_{1}=\{\emptyset\}, \alpha_{2}=\{\{\phi\}\}$ and $\alpha_{3}=$ $\{\{\phi\}, \emptyset\}$. In the other direction, we can encode $\mathcal{F}(\mathcal{M})$ in the standard modal language using

$$
\nabla_{\Sigma} \mathcal{X} \equiv \bigwedge_{\alpha \in \mathcal{X}} \square \bigvee_{\Phi \in \alpha} \widehat{\Phi} \wedge \bigwedge_{\Phi \in \operatorname{ch}(\mathcal{X})} \diamond \bigvee_{\alpha \in \mathcal{X}} \widehat{\Phi(\alpha)}
$$

where $\widehat{\Psi}=\bigwedge \Psi \wedge \neg \bigvee(\Sigma \backslash \Psi)$ for a finite set $\Psi \subseteq \mathcal{F}(T)$. In other words, both languages are expressively equivalent:

Proposition 4 (Translatability for Monotone Neighbourhood Models). The languages $\mathcal{M L}$ and $\mathcal{F}(\mathcal{M})$ can be effectively translated into one another, that is, there are computable functions $s: \mathcal{M L} \rightarrow \mathcal{F}(\mathcal{M})$ and $t: \mathcal{F}(\mathcal{M}) \rightarrow \mathcal{M L}$ such that

$\mathbb{C}, c|=\phi \Longleftrightarrow \mathbb{C}, c|=s(\phi)$ and $\mathbb{C}, c \mid \psi \Longleftrightarrow \mathbb{C}, c=t(\psi)$

for all $\mathcal{M}$-models $\mathbb{C}=(C, \gamma, \pi)$, all $c \in C$ and all $\phi \in \mathcal{F}(\mathcal{M})$ and $\psi \in \mathcal{M L}$.

Both for Kripke models and monotone neighbourhood models we see a strong correspondence between the logic of exact covers and the standard formalisms. We note in passing that a large number of frame classes arise as $T$-coalgebras for appropriate endofunctors $T$. This includes, other than Kripke frames and monotone neighbourhood frames, the class of not necessarily monotone neighbourhood frames, probabilistic frames and various classes of conditional frames. The paper [39] provides detail on all these examples. In the following sections, we study the logic of exact covers in general, that is, for an arbitrary (inclusion-preserving) endofunctor $T:$ Set $\rightarrow$ Set.

\section{The Hennessy-Milner Property}

The Hennessy-Milner property was one of the first results on coalgebraic logic, established by Moss in the first paper on the subject [26]. As we now show, the same can be said for the logic of exact covers. For the entire section, and indeed for the remainder of the paper, we work with an arbitrary inclusion-preserving endofunctor $T:$ Set $\rightarrow$ Set (not assuming that $T$ preserves weak pullbacks). To facilitate reasoning about bisimulation quantifiers later, we work with a notion of behavioural equivalence relative to a subset $\mathcal{V}_{0} \subseteq \mathcal{V}$ of propositional variables.

Definition 5 (Behavioural and Logical Equivalence). Suppose $\mathbb{C}=(C, \gamma, \pi)$ and $\mathbb{D}=(D, \delta, \sigma)$ are $T$-models and $\mathcal{V}_{0} \subseteq \mathcal{V}$ is a set of propositional variables. Two worlds $c \in C$ and $d \in D$ are $\mathcal{V}_{0}$-behaviourally equivalent, in symbols $c \simeq \mathcal{V}_{0} d$ if there exists a T-model $\mathbb{E}=(E, \epsilon, \tau)$ and two $\mathcal{V}_{0}$-morphisms $f: C \rightarrow E$ and $g: D \rightarrow E$ such that $f(c)=g(d)$. They are $\mathcal{V}_{0}$-logically equivalent, written $c \sim \mathcal{V}_{0} d$, if $\mathbb{C}, c=\phi \Longleftrightarrow$ $\mathbb{D}, d=\phi$ for all $\phi \in \mathcal{F}_{\mathcal{V}_{0}}(T)$. For $\mathcal{V}_{0}=\mathcal{V}$ we simply speak of behavioural $(\simeq)$ and logical $(\sim)$ equivalence.

Remark 6. If we take the underlying frame of a T-model $(C, \gamma, \pi)$ to be the T-coalgebra $(C, \gamma)$ and $\mathcal{V}_{0}=\emptyset$ then the notion of $\mathcal{V}_{0}$-behavioural equivalence coincides with with behavioural equivalence between the underlying frames.

It is easy to see that the logic of exact covers is compatible with morphisms of models.

Proposition 7 (Morphisms preserve Semantics). Let $\mathcal{V}_{0} \subseteq \mathcal{V}$ and suppose that $f: \mathbb{C} \rightarrow \mathbb{D}$ is a $\mathcal{V}_{0}$-morphism between two $T$-models $\mathbb{C}=(C, \gamma, \pi)$ and $\mathbb{D}=(D, \delta, \tau)$. Then $\mathbb{C}, c \models$ $\phi \Longleftrightarrow \mathbb{D}, f(c) \models \phi$ for all $c \in C$ and all $\phi \in \mathcal{F}_{\mathcal{V}_{0}}(T)$.

By the last proposition, invariance under behavioural equivalence is an easy consequence.

Corollary 8 (Invariance under Behavioural Equivalence). Let $\mathcal{V}_{0} \subseteq \mathcal{V}$ and suppose that $(C, \gamma, \pi)$ and $(D, \delta, \tau)$ are T-models and $c \in C, d \in D$ are $\mathcal{V}_{0}$-behaviourally equivalent. Then $\mathbb{C}, c \models \phi \Longleftrightarrow \mathbb{D}, d=\phi$ for all $\phi \in \mathcal{F}_{\mathcal{V}_{0}}(T)$.

Our goal for this section is to establish a converse of the above proposition in case $T$ is finitary. The proof strategy follows [37] and we show that the projection of an arbitrary model onto its quotient by logical equivalence is a morphism of models.

Lemma 9 (Quotient Models). Suppose that $\mathbb{C}=(C, \gamma, \pi) \in$ $\operatorname{Mod}(T)$ is a T-model and write $\sim$ for the relation of logical equivalence on $\mathbb{C}$. Then the maps

- $[\gamma]: C / \sim \rightarrow T(C / \sim)$ given by $[c] \mapsto T p \circ \gamma(c)$

- $[\pi]: C / \sim \rightarrow \mathcal{P}(\mathcal{V})$ given by $[c] \mapsto \pi(c)$

are well-defined. Moreover the canonical projection $p: C \rightarrow$ $C / \sim$ is a morphism of models $\mathbb{C} \rightarrow \mathbb{C} / \sim$ where $\mathbb{C} / \sim=(C / \sim$ $,[\gamma],[\pi])$ is the quotient model of $\mathbb{C}$.

This puts us into the position of characterising behavioural equivalence as follows:

Proposition 10 (Characterisation of Behavioural Equivalence). Suppose that $T$ : Set $\rightarrow$ Set is finitary. Then, for any $\mathbb{C}=$ $(C, \gamma, \pi)$ and $\mathbb{D}=(D, \delta, \tau)$ we have

$$
c \simeq d \Longleftrightarrow c \sim c^{\prime}
$$

for all $c \in C$ and all $d \in D$. 


\section{The Small Model Property}

We continue our investigation into the logic of exact covers by establishing the small model property: every satisfiable formula is satisfiable in a model of at most exponential size. Our treatment of the small model property is similar in spirit to [39] where small models are constructed using an inference system whereas we replace consistent sets of formulae by satisfiable sets. We use the following standard notions that allow us to build models from satisfiable sets of formulae.

Definition 11 (Subformulas and Closed Sets). The subformulae $\operatorname{subf}(\phi)$ of a formula $\phi \in \mathcal{F}(T)$ are defined inductively in the usual way where $\operatorname{subf}\left(\nabla_{\Sigma}(\mathcal{X})\right)=\left\{\nabla_{\Sigma}(\mathcal{X})\right\} \cup \bigcup\{\operatorname{subf}(\phi) \mid$ $\phi \in \Sigma\}$. The normalised negation $\sim \phi$ of a formula $\phi \in \mathcal{F}(T)$ is defined by $\sim(\neg \phi)=\phi$ and $\sim(\phi)=\neg \phi$, otherwise. A set $\Delta \subseteq \mathcal{F}(T)$ is closed if $\operatorname{subf}(\phi) \subseteq \Delta$ and $\sim \phi \in \Delta$ for all $\phi \in \Delta$.

Definition 12 (Theories and witnesses). Suppose $\Delta \subseteq \mathcal{F}(T)$ is closed. A subset $\Theta \subseteq \Delta$ is a $\Delta$-theory if there exists a $T$-model $\mathbb{C}=(C, \gamma, \pi)$ and $c \in C$ such that $\Theta=\{\phi \in \Delta \mid$ $\mathbb{C}, c \mid=\phi\}$. The pointed model $(\mathbb{C}, c)$ is then called a witness of theoryhood of $\Theta$.

Definition 13 (Coherent Models). Suppose $\Delta \subseteq \mathcal{F}_{\mathcal{V}}(T)$ is closed. A model $\mathbb{C}=(C, \gamma, \pi)$ is $\Delta$-coherent if

- $C=\{\Theta \subseteq \Delta \mid \Theta$ is a $\Delta$-theory $\}$ and, for all $\Theta \in C$,

- $p \in \Theta \Longleftrightarrow p \in \pi(\Theta)$ for all $p \in \mathcal{V}$

- $\nabla_{\Sigma}(\mathcal{X}) \in \Theta \Longleftrightarrow \operatorname{Tr}_{\Sigma} \circ \gamma(c)=\mathcal{X}$ for all $\nabla_{\Sigma}(\mathcal{X}) \in \Delta$ where $r_{\Sigma}: C \rightarrow \mathcal{P}(\Sigma)$ is the restriction map $r_{\Sigma}(\Theta)=\Theta \cap \Sigma$.

The small model property is now established by observing that coherent models satisfy the truth lemma and then later, that coherent models do indeed exist.

Lemma 14 (Truth Lemma). Suppose that $\Delta$ is closed and $\mathbb{C}=(C, \gamma, \pi)$ is $\Delta$-coherent. Then

$$
\mathbb{C}, \Theta \mid=\phi \Longleftrightarrow \phi \in \Theta
$$

for all $\Theta \in C$ and all $\phi \in \Delta$.

Lemma 15 (Existence Lemma). Suppose that $\Delta \subseteq \mathcal{F}_{\mathcal{V}}(T)$ is closed. Then a $\Delta$-coherent model exists.

The small model property is now an easy consequence of truth lemma and existence lemma.

Proposition 16 (Small Model Property). Suppose that $\phi \in$ $\mathcal{F}_{\mathcal{V}}(T)$ is satisfiable. Then there exists a model $\mathbb{C}=(C, \gamma, \pi)$ and $c \in C$ such that $|C| \leq 2^{2|\operatorname{subf}(\phi)|}$ and $\mathbb{C}, c \models \phi$.

\section{Tableaux Calculus and Distributive LaWs}

Distributive laws for coalgebraic logic [41] play a major role in the completeness of coalgebraic logic [18] (indeed, distributivity over conjunctions is one of the key derivation rules). In [36], distributive laws for monotone modal logic have been used to establish uniform interpolation. Here, we establish two distributive laws for the logic of exact covers: we show that $\nabla$ distributes over both conjunction and negation which forms the basis of a complete tableaux calculus.
Proposition 17 (Distributivity over Conjunctions). Let $\mathbb{C}=$ $(C, \gamma, \pi) \in \operatorname{Mod}_{\mathcal{V}}(T)$ and $\nabla_{\Sigma_{2}}\left(\mathcal{X}_{1}\right), \nabla_{\Sigma_{2}}\left(\mathcal{X}_{2}\right) \in \mathcal{F}$. Then, for $\Sigma=\Sigma_{1} \cup \Sigma_{2}$, and all $c \in C$

$$
\mathbb{C}, c \models \nabla_{\Sigma_{1}}\left(\mathcal{X}_{1}\right) \wedge \nabla_{\Sigma_{2}}\left(\mathcal{X}_{2}\right) \Longleftrightarrow \mathbb{C}, c \models \nabla_{\Sigma}(\mathcal{X})
$$

for some $\mathcal{X} \in \mathcal{R}_{\mathcal{X}_{2}}^{\mathcal{X}_{1}}\left(\Sigma_{1}, \Sigma_{2}\right)$ where $\mathcal{R}_{\mathcal{X}_{2}}^{\mathcal{X}_{1}}\left(\Sigma_{1}, \Sigma_{2}\right)=\{\mathcal{X} \in$ $T \mathcal{P}(\Sigma) \mid \operatorname{Tr}_{i}(\mathcal{X})=\mathcal{X}_{i}$ for $\left.i=1,2\right\}$ and $r_{i}: \mathcal{P}(\Sigma) \rightarrow \mathcal{P}\left(\Sigma_{i}\right)$ is the restriction map $r_{i}(\Phi)=\Phi \cap \Sigma_{i}$.

In case $T$ preserves finite sets, this gives the following logical distributive law.

Corollary 18 (Distributivity over Conjunction Law). Suppose $T:$ Set $\rightarrow$ Set maps finite sets to finite sets. Then

$\nabla_{\Sigma_{1}}\left(\mathcal{X}_{1}\right) \wedge \nabla_{\Sigma_{2}}\left(\mathcal{X}_{2}\right) \equiv \bigvee\left\{\nabla_{\Sigma_{1} \cup \Sigma_{2}}(\mathcal{X}) \mid \mathcal{X} \in \mathcal{R}_{\mathcal{X}_{2}}^{\mathcal{X}_{1}}\left(\Sigma_{1}, \Sigma_{2}\right)\right\}$ for all formulae $\nabla_{\Sigma_{1}}\left(\mathcal{X}_{1}\right)$ and $\nabla_{\Sigma_{2}}\left(\mathcal{X}_{2}\right)$.

We now turn to distributivity of $\nabla$ over negations where the central idea is that every world satisfies a $\nabla$-formula and we need to select accordingly.

Proposition 19 (Distributivity over Negation). Let $\mathbb{C}=$ $(C, \gamma, \pi) \in \operatorname{Mod}(T)$ and $\nabla_{\Sigma}(\mathcal{X}) \in \mathcal{F}(T)$. Then

$\mathbb{C}, c \mid \neg \nabla_{\Sigma}(\mathcal{X}) \Longleftrightarrow \mathbb{C}, c \models \nabla_{\Sigma}(\mathcal{Y})$ for some $\mathcal{Y} \in \mathcal{N}_{\Sigma}(\mathcal{X})$ where $\mathcal{N}_{\Sigma}(\mathcal{X})=\{\mathcal{Y} \in T \mathcal{P}(\Sigma) \mid \mathcal{Y} \neq \mathcal{X}\}$ are the possible negations of $\mathcal{X}$.

Again this gives a logical distributive law for functors that preserve finite sets.

Corollary 20 (Distributivity over Negation). Suppose T maps finite sets to finite sets. Then

$$
\neg \nabla_{\Sigma}(\mathcal{X}) \equiv \bigvee\left\{\nabla_{\Sigma}(\mathcal{Y}) \mid \mathcal{Y} \in \mathcal{N}_{\Sigma}(\mathcal{X})\right\}
$$

for all formulae $\nabla_{\Sigma} \in \mathcal{F}(T)$.

Distributivity over negation and conjunction form the basis for a complete tableaux calculus for the logic of exact covers. The remarkable feature of this calculus is that all rules are invertible. Our tableaux are set-labelled tableaux and the existence of a closed tableau is equivalent to the unsatisfiability of the root. The crucial ingredient in the calculus is the following characterisation of the satisfiability of $\nabla$-formulae. We introduce terminology following [21, Section 5] for standard functors.

Definition 21. If $\Sigma \subseteq \mathcal{F}(T)$ is finite and $\mathcal{X} \in T \mathcal{P}(\Sigma)$,

$$
\operatorname{Base}(\mathcal{X})=\bigcap\{\alpha \subseteq \mathcal{P}(\Sigma) \mid \mathcal{X} \in T \alpha\}
$$

is called the base of $\mathcal{X}$.

It is known that $\mathcal{X} \in \operatorname{Base}(\mathcal{X})$ and moreover $\operatorname{Base}(\mathcal{X})$ is the smallest subset of $\mathcal{P}(\Sigma)$ with this property [21, Proposition 6.7]. Using the notion of base, we can characterise satisfiability of $\nabla$-formulae as follows.

Proposition 22. Let $\nabla_{\Sigma}(\mathcal{X}) \in \mathcal{F}(T)$ and $P \subseteq \mathcal{V} \cup \neg \mathcal{V}$ be finite. Then $\nabla_{\Sigma}(\mathcal{X}) \wedge \wedge P$ is satisfiable iff $\wedge P$ is satisfiable and $\wedge \Phi \wedge \neg \bigvee(\Sigma \backslash \Phi)$ is satisfiable for all $\Phi \in \operatorname{Base}(\mathcal{X})$. 
Together with the distributive laws we obtain the following tableau calculus, formulated over multisets.

Definition 23 (Tableau Calculus). A sequent is a finite multiset of formulae in $\mathcal{F}(T)$. We use Greek letters $\Gamma, \Delta$ for sequents and write $\Gamma, \Delta$ for the multiset union of $\Gamma$ and $\Delta$ and identify formulae with singleton multisets. The tableau calculus for the logic of exact covers contains the propositional rules

$$
(\mathrm{Ax}) \frac{p, \neg p, \Gamma}{\Gamma, \phi, \psi} \quad(\wedge) \frac{\Gamma, \phi \wedge \psi}{\Gamma} \quad(\vee) \frac{\Gamma, \phi \vee \psi}{\Gamma, \phi \quad \Gamma, \psi}
$$

together with the following rules for $\nabla$

$$
\begin{gathered}
(\wedge \nabla) \frac{\nabla_{\Sigma_{1}}\left(\mathcal{X}_{1}\right), \nabla_{\Sigma_{2}}\left(\mathcal{X}_{2}\right), \Gamma}{\left\{\nabla_{\Sigma_{1} \cup \Sigma_{2}}(\mathcal{X}), \Gamma \mid \mathcal{X} \in \mathcal{R}_{\mathcal{X}_{2}}^{\mathcal{X}_{1}}\left(\Sigma_{1}, \Sigma_{2}\right)\right\}} \\
(\neg \nabla) \frac{\neg \nabla_{\Sigma}(\mathcal{X}), \Gamma}{\left\{\nabla_{\Sigma}(\mathcal{Y}), \Gamma \mid \mathcal{Y} \in \mathcal{N}_{\Sigma}(\mathcal{X})\right\}} \\
(\nabla) \frac{\nabla_{\Sigma}(\mathcal{X}), \Gamma}{\Phi \cup\{\neg \psi \mid \psi \in \Sigma \backslash \Phi\}}(\Phi \in \operatorname{Base}(\mathcal{X}))
\end{gathered}
$$

where the last rule is subject to the side condition that no other rule is applicable, i.e. $\Gamma \subseteq \mathcal{V} \cup \neg \mathcal{V}$ and $\Gamma \cap \neg \Gamma=\emptyset$. Note that unless $T$ preserves finite sets the above rules may have an infinite number of conclusions. A tableau is a tree constructed according to the rules above, and a tableau is closed if all its leaves are labelled with $(\mathrm{Ax})$.

Our first observation is that all branches in tableaux are finite.

Lemma 24 (All Tableaux are Finite). All tableaux for the logic of exact covers only have branches of finite length.

Our goal is to show that a formula $\phi \in \mathcal{F}(T)$ is unsatisfiable iff there exists a closed tableau with root $\phi$. Our first result is invertibility:

Proposition 25 (Invertibility). Let $\Gamma \subseteq \mathcal{F}(T)$ be a sequent. Then $\Gamma$ is satisfiable iff, for all rules $\frac{\Gamma}{\left\{\Gamma_{i} i \in I\right\}}$ with premiss $\Gamma$ there exists $i \in I$ such that $\Gamma_{i}$ is satisfiable.

For completeness we are now in a position to argue in terms of invertibility.

Theorem 26 (Completenss). Let $\Gamma \subseteq \mathcal{F}(T)$ be a sequent. Then $\Gamma$ is satisfiable iff no closed tableau with root $\Gamma$ exists.

\section{Closure under Bisimulation Quantifiers}

One of the remarkable features of the logic of exact covers is closure under bisimulation quantifiers. As in [36], closure under bisimulation quantifiers immediately gives uniform interpolation not only for the logic of exact covers but also for other equipollent logics.

To establish closure under bisimulation quantifiers, we first show that every formula is equivalent to a (possibly empty) disjunction of satisfiable formulae of type $\nabla_{\Sigma}(\Phi) \wedge \wedge P$ where $P$ is a finite set of possibly negated propositional variables and $\Sigma$ is (inductively) a set of formulae of the same shape: that is, negation can be eliminated and conjunction is only necessary for specifying values of propositional variables. By induction on the structure of these structurally simpler formulae we can then show closure under bisimulation quantifiers, essentially because the latter distribute over disjunctions and interact with functor application. The formal notions are as follows:

Definition 27 (Essential Formulae and Bisimulation Quantifiers). Suppose that $\mathcal{V}_{0} \subseteq \mathcal{V}$ is a set of propositional variables. The essential formulae over $\mathcal{V}_{0}$ are given by

$$
\mathcal{E}_{\mathcal{V}_{0}}(T)::=\nabla_{\Sigma}(\mathcal{X}) \wedge \bigwedge P \mid \bigvee \Phi
$$

where all of $\Sigma \subseteq \mathcal{E}_{\mathcal{V}_{0}}(T), P \subseteq \mathcal{V}_{0} \cup \neg \mathcal{V}_{0}$ and $\Phi \subseteq \mathcal{E}_{\mathcal{V}_{0}}(T)$ are finite, $\mathcal{X} \in T \mathcal{P}(\Sigma)$ and $\nabla_{\Sigma}(\mathcal{X}) \wedge \wedge P$ is satisfiable. As for $\mathcal{F}(T)$ we write $\mathcal{E}(T)$ for $\mathcal{E}_{\mathcal{V}}(T)$. The bisimulation quantifier extension $\exists \mathcal{F}_{\mathcal{V}_{0}}(T)$ of the language of exact covers (and, respectively, its essential fragment $\exists \mathcal{E}_{\mathcal{V}_{0}}(T)$ ) is the least set of formulae that contains $\mathcal{F}_{\mathcal{V}_{0}}(T)$ (respectively $\mathcal{E}_{\mathcal{V}_{0}}(T)$ ) and the formula $\exists p . \phi$ for every $p \in \mathcal{V}_{0}$ and every $\phi \in \mathcal{F}_{\mathcal{V}_{0}}(T)$ (respectively $\phi \in \mathcal{E}_{\mathcal{V}_{0}}(T)$ ). If $\mathbb{C}=(C, \gamma, \pi)$ is a $T$-model, we extend the satisfaction relation by defining

$$
\begin{aligned}
& \mathbb{C}, c \mid=\exists p . \phi \Longleftrightarrow \\
& \quad \mathbb{C}^{\prime}, c^{\prime}=\phi \text { for some } \mathbb{C}^{\prime}, c^{\prime} \text { such that } \mathbb{C}^{\prime}, c^{\prime} \simeq \mathcal{V} \backslash\{p\} \mathbb{C}, c
\end{aligned}
$$

for $c \in C$ and a propositional variable $p \in \mathcal{V}$.

Remark 28. The notion of essential formulae introduced above is closely related to that of transparent formulae used in [36]. We have chosen different terminology to avoid confusion and think of unsatisfiable formulae of the form $\nabla_{\Sigma}(\mathcal{X}) \wedge \wedge P$ as not being essential. Note that not every essential formula is satisfiable: a counterexample is provided by $\bigvee \emptyset$.

It follows from Corollaries 18 and 20 that every formula is equivalent to an essential formula in the same variables. The proof requires us to translate formulae of the form $\nabla_{\Sigma}(\mathcal{X})$ into essential formulae which is catered for by the following preliminary lemma that we also use to show that $\mathcal{E}(T)$ is closed under bisimulation quantifiers.

Lemma 29. Suppose that $\Sigma \subseteq \exists \mathcal{F}(T)$ is finite and $f: \Sigma \rightarrow$ $\exists \mathcal{F}(T)$ is a function such that $\phi \equiv f(\phi)$ for all $\phi \in \Sigma$. Then

$$
\nabla_{\Sigma}(\mathcal{X}) \equiv \nabla_{f[\Sigma]}(T \mathcal{P} f(\mathcal{X}))
$$

for all $\mathcal{X} \in T \mathcal{P}(\Sigma)$ for which $\nabla_{\Sigma}(\mathcal{X})$ is satisfiable.

Using the above lemma we can now show that every formula is equivalent to a essential formula (and later that the fragment of essential formulae is closed under bisimulation quantifiers).

Proposition 30 (Equivalence to Essential Formulae). Suppose that $T$ preserves finite sets. Then there exists a function $d$ : $\mathcal{F}_{\mathcal{V}_{0}}(T) \rightarrow \mathcal{E}_{\mathcal{V}_{0}}(T)$ such that $d(\phi) \equiv \phi$ for all $\phi \in \mathcal{F}_{\mathcal{V}_{0}}(T)$.

Our next goal is to show that $\mathcal{E}(T)$, and hence by the previous Proposition also $\mathcal{F}(T)$, is closed under bisimulation quantifiers, where we abuse terminology slightly in that we use (coalgebraic) behavioural equivalence, rather than (coalgebraic) bisimulation (Definition 5) as the defining notion of equivalence. 
As bisimulation quantifiers distribute over disjunctions, it is easy to see that the essential fragment $\mathcal{E}(T)$ of the logic of exact covers is closed under bisimulation quantifiers. The key lemma is the following:

Lemma 31 (Elimination of Bisimulation Quantifiers). Suppose that $\mathcal{V}_{0} \subseteq \mathcal{V}$ is a (not necessarily finite) set of propositional variables. Then, for every $\phi \in \mathcal{F}_{\mathcal{V}_{0}}(T)$ and every $p \in \mathcal{V}_{0}$ there exists a formula $e_{p}(\phi) \in \mathcal{E}_{\mathcal{V}_{0} \backslash\{p\}}(T)$ such that $\exists p . \phi \equiv e_{p}(\phi)$.

Repeated application of the previous lemma gives closure under bisimulation quantifiers for the logic of exact covers.

Theorem 32 (Closure under Bisimulation Quantifiers). Suppose that $T$ preserves finite sets. Then, for all formulae $\phi \in \exists \mathcal{F}(T)$ there is a formula $q(\phi) \in \mathcal{F}(T)$ such that $q(\phi) \equiv \phi$

As an application, we show that the logic of exact covers has uniform interpolation. We present the result in detail to demonstrate that the standard argument [34], [11] smoothly translates to our setting.

Theorem 33 (Uniform Interpolation). Suppose that $\mathcal{V}_{0}, \mathcal{V}_{1}$ and $\mathcal{V}_{2} \subseteq \mathcal{V}$ are finite sets of propositional variables with $\mathcal{V}_{0} \cap \mathcal{V}_{1} \subseteq \mathcal{V}_{2}$. Then, for all $\phi \in \mathcal{F}_{\mathcal{V}_{0}}(T)$ there exists $i(\phi) \in$ $\mathcal{F}_{\mathcal{V}_{2}}(T)$ such that $\models \phi \rightarrow \psi \Longleftrightarrow \models i(\phi) \rightarrow \psi$ for all $\psi \in \mathcal{F}\left(\mathcal{V}_{1}\right)$.

As corollaries, we obtain uniform interpolation and closure under bisimulation quantifiers for all logics that are equiexpressive to the logic of exact covers which includes monotone modal logic and the logic $\mathrm{K}$ by Propositions 3 and 4 . Rather than encoding particular logics into the logic of exact covers in a case-by-case manner, we now show that the same results can also be obtained uniformly for all rank-1 logics

\section{Applications to Rank-1 Logics}

We now turn our attention to modal logics in the standard sense (extensions of classical propositional calculus with modal operators). Our main result is that every rank-1 logic is equipollent to the logic of exact covers which allows us to transfer results back and forth. Concrete applications then give interpolation theorems for, amongst others, the modal logics $\mathrm{K}$, classical and monotone modal logic, graded and probabilistic modal logic and alternating temporal logic.

Definition 34 (Rank-1 Axioms and Rank-1 Rules). Let $\Lambda$ be a modal similarity type. A rule is of the form $\phi / \psi$ where $\phi, \psi \in \mathcal{F}(\Lambda)$. The rule $\phi / \psi$ is rank-1 if $\phi \in \operatorname{Prop}(\mathcal{V})$ and $\psi \in \operatorname{Prop}(\Lambda(\mathcal{V}))$. A rule $\phi / \psi$ is admissible in a logic $\mathcal{L}$ if, for all substitutions $\sigma: \mathcal{V} \rightarrow \mathcal{F}(\Lambda)$, we have that $\psi \sigma \in \mathcal{L}$ whenever $\phi \sigma \in \mathcal{L}$. We write $\mathcal{R}_{1}(\mathcal{L})$ for the set of rank-1 rules that are admissible in $\mathcal{L}$. A rank-1 axiom is a formula $\phi \in \operatorname{Prop}(\Lambda(\operatorname{Prop}(\phi)))$ and we write $\mathcal{A}_{1}(\mathcal{L})$ for the set of rank-1 axioms contained in $\mathcal{L}$. If $A$ is a set of rank-1 axioms ( $R$ is a set of rank-1 rules), we write $\mathcal{L}^{\Lambda}(A)\left(\mathcal{L}^{\Lambda}(R)\right.$ ) for the smallest $\Lambda$-logic that contains $A$ (for which all $\phi / \psi \in R$ are admissible) and drop the superscript ' $\Lambda$ ' denoting the similarity type if it is clear from the context.

Remark 35. Several different notions of rank-1 rules have been used in the literature. In [39] a one-step rule is taken to be of the form $\phi / \psi$ where $\phi$ is propositional and $\psi$ is a clause over $\Lambda(\mathcal{V})$ which is equivalent to the notion of rank-1 rule used here modulo converting the conclusion into conjunctive normal form. In the context of sequent calculi [31] one-step rules are taken to consist of propositional premises with conclusions in $\Lambda(\mathcal{V}) \cup \neg \Lambda(\mathcal{V})$ which is again equivalent to the notion of onestep rule here modulo converting both premiss and conclusion to conjunctive normal form.

We note the following basic facts. The proof of (iv) uses the notion of projectivity introduced in [13] and is crucial for strong completeness..

Lemma 36 (Consistency and Admissibility). Let $L$ be a $\Lambda$ logic and $\phi / \psi$ a $\Lambda$-rule with $\phi \in \operatorname{Prop}(\mathcal{V})$.

(i) $\mathcal{L}\left(\mathcal{R}_{1}(L)\right) \subseteq L$ and $\mathcal{L}\left(\mathcal{A}_{1}(L)\right) \subseteq L$.

(ii) Both $\mathcal{L}\left(\mathcal{R}_{1}\left(\mathcal{L}\left(\mathcal{R}_{1}(L)\right)\right)=\mathcal{L}\left(\mathcal{R}_{1}(L)\right)\right.$ and $\mathcal{L}\left(\mathcal{A}_{1}\left(\mathcal{L}\left(\mathcal{A}_{1}(L)\right)\right)\right)=\mathcal{L}\left(\mathcal{A}_{1}(L)\right)$.

(iii) $L$ is consistent iff $L \vdash \phi \Longleftrightarrow \mathrm{PL} \vdash \phi$ for all $\phi \in$ $\operatorname{Prop}(\mathcal{V})$.

(iv) $\phi / \psi$ is $L$-admissible iff $\psi \sigma \in L$ for all $L$-unifiers $\sigma$ : $\mathcal{V} \rightarrow \operatorname{Prop}(\mathcal{V})$ of $\phi$

We will focus on so-called rank-1 logics in the sequel which can be characterised by any of the two following conditions the equivalence of which is implicit in [39, Proposition 15].

Lemma and Definition 37 (Rank-1 Logics). Let $\Lambda$ be a similarity type. Then $\mathcal{L}\left(\mathcal{A}_{1}(L)\right)=\mathcal{L}\left(\mathcal{R}_{1}(L)\right)$ for every $\Lambda$-logic $L$. We say that $L$ is rank-1 if $L=\mathcal{L}\left(\mathcal{A}_{1}(L)\right)$, or equivalently, $L=\mathcal{L}\left(\mathcal{R}_{1}(L)\right)$.

In other words, rank-1 logics are determined by the set of their admissible one-step rules or equivalently, by the set of one-step axioms they contain. Note that a rank-1 axiom is a propositional combination of atoms of the form $\nabla\left(\phi_{1}, \ldots, \phi_{n}\right)$ where $\phi_{1}, \ldots, \phi_{n}$ are propositional. In particular, every rank1 logic is non-iterative in the sense of Lewis [24] but the converse is not necessarily true: a counterexample is the modal $\operatorname{logic} T$ of reflexive frames, the axiomatisation of which relies on the (reflexivity) axiom $\square p \rightarrow p$. Nonetheless, we have a large range of examples.

Example 38 (Rank-1 Logics). The class of rank-1 logics contains the modal logics $\mathrm{K}$ and $\mathrm{KD}$, classical modal logic $\mathrm{E}$ and monotone modal logic $\mathrm{M}$ and basic conditional logic CK all of which are discussed in [8]. Moreover, the class of rank-1 logics subsumes Pauly's coalition logic [33] (which independently appears as alternating-time temporal logic in [3]). It also comprises graded [10] and probabilistic [22], [16] modal logic and their extensions with linear inequalities [19] as well as majority logic [29] and all extensions of the above by rank-1 axioms, for example Burgess' system $\mathcal{S}$ [7] that implements the KLM-axioms [17] in conditional logic. 
Also note that any modal logic that is axiomatised by rank-1 axioms or rules is automatically a rank-1 logic in the sense of Definition 37 and all logics that are amenable to coalgebraic semantics are rank-1, see [39] for examples.

It has been observed in [38] that every rank-1 logic can be equipped with a sound and complete coalgebraic semantics which allows us to transfer interpolation from the logic of exact covers (given by coalgebraic semantics) to any given rank-1 logic. We repeat this construction here for the reader's convenience.

Definition 39 (Coalgebraic Semantics). Let $L$ be a rank-1 logic. The functor induced by $L$ is given by $T_{L}$ : Set $\rightarrow$ Set by $T_{L}(X)=\operatorname{Uf}(F \Lambda(\mathcal{P} X) / \sim)$ where $\sim$ is the congruence generated by $\left\{\psi \sigma=\top \mid \phi / \psi \in \mathcal{R}_{1}(L), \sigma: \mathcal{V} \rightarrow\right.$ $\mathcal{P}(X)$ and $\phi \sigma=\top\}$. To every $n$-ary $\varnothing \in \Lambda$ we associate a natural transformation $\llbracket \lambda \rrbracket: \mathcal{Q}^{n} \rightarrow \mathcal{Q} \circ T^{\mathrm{op}}$ given by $\llbracket \nabla \rrbracket_{X}\left(A_{1}, \ldots, A_{n}\right)=\left\{u \in T_{L} X \mid \nabla\left(A_{1}, \ldots, A_{n}\right) \in u\right\}$. We obtain an interpretation of $\mathcal{F}(\Lambda)$ over T-models $\mathbb{C}=(C, \gamma, \pi)$ from the usual clauses for the propositional connectives, together with

$\mathbb{C}, c \models \varnothing\left(\phi_{1}, \ldots, \phi_{n}\right) \Longleftrightarrow \gamma(c) \in \llbracket \nabla \rrbracket_{C}\left(\llbracket \phi_{1} \rrbracket_{\mathbb{C}}, \ldots, \llbracket \phi_{n} \rrbracket_{\mathbb{C}}\right)$

where $\llbracket \phi \rrbracket_{\mathbb{C}}=\{c \in C|\mathbb{C}, c|=\phi\}$ is the truth-set of $\phi$. We write $L \models \phi$ if $\mathbb{C}, c \models \phi$ for all $T_{L}$-models $\mathbb{C}=(C, \gamma, \pi)$ and all $c \in C$.

To make sense of the definition of $T_{L}$, note that $\phi \sigma$ and $\psi \sigma$ are terms over the boolean algebras $\mathcal{P}(X)$ and $F \Lambda(\mathcal{P}(X))$, respectively.

Remark 40. The above definition is an instance of coalgebraic semantics of modal logics in terms of predicate liftings, introduced in [32] (see [20] for an overview) but specialised to the functor $T_{L}$ induced by a rank-1 logic.

While the semantics introduced above is arguably synthetic and not the natural semantics for the rank-1 logics of Example 38 it still serves the main purpose of the present paper. We refer the reader to [39], [20] for an overview of coalgebraic logics and their (natural) interpretations. The main result of [38] is soundness and completeness of rank-1 logics $L$ over $T_{L}$-models. Here, we need a formulation of completeness that is slightly stronger than that provided in op.cit: : completeness over $T_{L}$-models also obtains for sub-logics of $L$ that arise by restricting to a subset of modal operators.

Theorem 41 (Strong Completeness over Restricted Similarity Types). Let $L$ be a rank-1 logic over a (not necessarily finite) similarity type $\Lambda$ and let $\Lambda_{0} \subseteq \Lambda$ be a (not necessarily finite) sub-similarity type. Then the following are equivalent:

(i) $\Phi$ is $\mathcal{L}^{\Lambda_{0}}\left(\left\{\phi / \psi \in \mathcal{R}_{1}(L) \mid \psi \in \mathcal{F}\left(\Lambda_{0}\right)\right\}\right)$-consistent.

(ii) $\Phi$ is satisfiable in $\operatorname{Mod}\left(T_{L}\right)$.

for all (not necessarily finite) sets $\Phi \subseteq \mathcal{F}(\Lambda)$.

The completeness theorem claimed in [38] now arises as a consequence of the previous theorem. In what follows, we only need weak completeness, i.e. the following immediate corollary that can alternatively be inferred from completeness of $L$ with respect to $T_{L}$-models [38] and the fact that all onestep sound one-step rules are one-step complete [39].

Corollary 42 (Completeness over Restricted Similarity Types). Under the assumptions of Theorem 41 we have that

$$
\phi \in \mathcal{L}^{\Lambda_{0}}\left(\left\{\phi / \psi \in \mathcal{R}_{1}(L) \mid \psi \in \mathcal{F}\left(\Lambda_{0}\right)\right\}\right) \Longleftrightarrow L \models \phi
$$

and in particular $L \vdash \phi \Longleftrightarrow L \models \phi$ for all $\phi \in \mathcal{F}\left(\Lambda_{0}\right)$.

We now translate between a rank-1 logic $L$ and the logic of exact covers induced by $T_{L}$ where $T_{L}$-models provide the notion of semantical equivalence. We pause briefly to better understand the nature of the logic of exact covers when instantiated to the functor $T_{L}$ given by a rank-1 logic $L$.

Remark 43. Let $L$ be rank-1 with induced functor $T_{L}$.

(i) The transition map $\gamma: C \rightarrow T_{L} C$ of a $T_{L}$-model takes values in $\operatorname{Uf}(F \Lambda(\mathcal{P}(C)) / \sim)$, that is, each $\gamma(c)$ is an ultrafilter containing $\sim$-equivalence classes $[\phi]$ where $\phi \in F \Lambda(\mathcal{P}(C))$ is an element of the free boolean algebra over generators $\nabla\left(A_{1}, \ldots, A_{n}\right)$ where $A_{1}, \ldots, A_{n} \subseteq C$. As a consequence, the ultrafilter $\gamma(c)$ is determined by its elements of the form $\left[\odot\left(A_{1}, \ldots, A_{n}\right)\right]$.

(ii) If $\Sigma \subseteq \mathcal{F}\left(T_{L}\right)$ is finite and $\mathcal{X} \in T_{L} \mathcal{P}(\Sigma)$ then $\mathcal{X}$ is an ultrafilter consisting of $\sim$-equivalence classes $\left[\nabla\left(\alpha_{1}, \ldots, \alpha_{n}\right)\right]$ where each $\alpha_{i} \in \mathcal{P}(\mathcal{P}(\Sigma))$ is an element of the double(!) powerset of $\Sigma$ as one can readily verify by unravelling the definitions. As above, this implies that every $\mathcal{X} \in T_{L} \mathcal{P}(\Sigma)$ is determined by its elements of the form $\left[\nabla\left(\alpha_{1}, \ldots, \alpha_{n}\right)\right]$.

The following lemma is the crucial stepping stone for the translation between $\mathcal{F}(\Lambda)$ and $\mathcal{F}\left(T_{L}\right)$.

Lemma 44. Let $L$ be a rank-1 logic over a finite similarity type $\Lambda$ and $\Sigma \subseteq \mathcal{F}\left(T_{L}\right)$ be finite and $\mathcal{X} \in T_{L} \mathcal{P}(\Sigma)$. If $\mathbb{C}=$ $(C, \gamma, \pi)$ is a $T_{L}$-model, then

$$
\mathbb{C}, c=\nabla_{\Sigma}(\mathcal{X}) \Longleftrightarrow \begin{gathered}
\bigwedge\left[\nabla \llbracket \alpha_{1} \rrbracket, \ldots, \llbracket \alpha_{n} \rrbracket\right] \wedge \\
{\left[\odot\left(\alpha_{1}, \ldots, \alpha_{n}\right)\right] \in \mathcal{X}} \\
\neg \bigvee\left[\odot\left(\llbracket \alpha_{1} \rrbracket, \ldots, \llbracket \alpha_{n} \rrbracket\right] \in \gamma(c)\right. \\
{\left[\odot\left(\alpha_{1}, \ldots, \alpha_{n}\right)\right] \notin \mathcal{X}}
\end{gathered}
$$

for all $c \in \mathbb{C}$ where $\llbracket \alpha \rrbracket=\{c \in C \mid \exists \Phi \in \alpha . c \models \bigwedge \Phi \wedge$ $\neg \bigvee(\Sigma \backslash \Phi)\}$ is the truth-set of $\alpha \in \mathcal{P} \mathcal{P}(\Sigma)$. Moreover, if $\phi_{1}, \ldots, \phi_{n} \in \mathcal{F}(\Lambda)$ then

$$
\mathbb{C}, c=\varnothing\left(\phi_{1}, \ldots, \phi_{n}\right) \Longleftrightarrow\left[\nabla\left(\llbracket \phi_{1} \rrbracket, \ldots, \llbracket \phi_{n} \rrbracket\right)\right] \in \gamma(c)
$$

where $\llbracket \phi \rrbracket=\{c \in C \mid \mathbb{C}, c \models \phi\}$ is the truth-set of $\phi \in \mathcal{F}(\Lambda)$.

The equipollence of $\mathcal{F}(\Lambda)$ and $\mathcal{F}\left(T_{L}\right)$ over $T_{L}$-models is now easily established over finite similarity types.

Proposition 45 (Back-and-Forth Translation for Finite $\Lambda$ ). Let $\Lambda$ be a finite similarity type and $L$ a rank-1 logic over $\Lambda$ and $\mathcal{V}_{0} \subseteq \mathcal{V}$. Then there are functions $b: \mathcal{F}_{\mathcal{V}_{0}}\left(T_{L}\right) \rightarrow \mathcal{F}_{\mathcal{V}_{0}}(\Lambda)$ and $f: \mathcal{F}_{\mathcal{V}_{0}}\left(T_{L}\right) \rightarrow \mathcal{F}_{\mathcal{V}_{0}}(\Lambda)$ such that

$$
b(\phi) \equiv \phi \text { and } f(\psi) \equiv \psi
$$


for all $\phi \in \mathcal{F}\left(T_{L}\right)$ and all $\psi \in \mathcal{F}(\Lambda)$.

The avid reader is invited to compare the above translations to the translations for Kripke frames and monotone neighbourhood frames given in Section III. In particular, we can now establish our first main theorem:

Theorem 46 (Uniform Interpolation for Finite Similarity Types). Suppose that L is a rank-1 logic over a finite similarity type $\Lambda$. Then $L$ has uniform interpolation. That is, for every $\phi \in \mathcal{F}_{\mathcal{V}_{0}}$ there exists $i(\phi) \in \mathcal{F}_{\mathcal{V}_{2}}(\Lambda)$ such that

$$
L \vdash \phi \rightarrow \psi \Longleftrightarrow L \vdash i(\phi) \rightarrow \psi
$$

for all $\psi \in \mathcal{F}_{\mathcal{V}_{1}}(\Lambda)$, where $\mathcal{V}_{0}, \mathcal{V}_{1}$ and $\mathcal{V}_{2}$ are finite sets of propositional variables with $\mathcal{V}_{0} \cap \mathcal{V}_{1} \subseteq \mathcal{V}_{2}$.

We can in fact obtain interpolation for all rank-1 logics irrespective of the size of the similarity type. This follows as restrictions of rank-1 logics are rank-1 themselves, the proof of which uses Corollary 42 .

Theorem 47 (Restrictions of Rank-1 Logics). Suppose that $L$ is a rank-1 logic over a (not necessarily finite) similarity type $\Lambda$ and $\Lambda_{0} \subseteq \Lambda$. Then $L \cap \mathcal{F}\left(\Lambda_{0}\right)$ is a rank-1 logic (over $\Lambda_{0}$ ).

Put into different words, if a formula $\phi \in \mathcal{F}\left(\Lambda_{0}\right)$ is provable using rules that are admissible in $L$, then it is already provable using rules that only mention $\Lambda_{0}$-modalities. We have given a semantic account of this fact, alternative proofs would establish cut-elimination and the subformula property. As a consequence, we have almost uniform interpolation for all rank-1 logics.

Theorem 48 (Interpolation for Infinite Similarity Types). Suppose that $L$ is a rank-1 logic over the modal similarity type $\Lambda$ and $\Lambda_{0} \subseteq \Lambda_{1}$ are finite sub-similarity types and let $\mathcal{V}_{0}, \mathcal{V}_{1}$ and $\mathcal{V}_{2}$ be finite sets of propositional variables with $\mathcal{V}_{0} \cap \mathcal{V}_{1} \subseteq \mathcal{V}_{2}$. Then, for all $\phi \in \mathcal{F}_{\mathcal{V}_{0}}\left(\Lambda_{0}\right)$ there exists $i(\phi) \in \mathcal{F}_{\mathcal{V}_{2}}\left(\Lambda_{1}\right)$ such that

$$
L \vdash \phi \rightarrow \psi \Longleftrightarrow L \vdash i(\phi) \rightarrow \psi
$$

for all $\psi \in \mathcal{F}_{\mathcal{V}_{1}}\left(\Lambda_{2}\right)$.

For the logics introduced in Example 38 the situation is as follows:

Example 49 (Uniform Interpolation for Rank-1 Logics). Applying Theorem 46 and Theorem 48 to the logics introduced in Example 38 we obtain the following interpolation theorems.

(i) We obtain uniform interpolation for the logics $\mathrm{K}$ and $\mathrm{KD}$, classical modal logic $\mathrm{E}$ and monotone modal logic $\mathrm{M}$ and basic conditional logic CK as well as Burgess' system $\mathcal{S}$ and alternating temporal logic over a finite number of agents, as the similarity type of all these logics are finite. With the exception of $\mathrm{K}$ and $\mathrm{M}$, these results appear to be new.

(ii) We obtain interpolation for the logics $\mathrm{G}$ and $\mathrm{P}$ as well as their extension with linear inequalities as the similarity type of these logics is infinite. These interpolation results appear to be new.
The assiduous reader will have noticed that both Theorem 46 and Theorem 48 do not state that interpolants can be computed effectively as is the case for all interpolation theorems in the literature. To guarantee this, we need to assume additionally that the logic under scrutiny is decidable itself.

Theorem 50 (Effective Computation of Interpolants). Suppose that $L$ is a decidable rank-1 logic. Then the interpolant $i(\phi)$ in Theorem 48 is effectively constructible from $\phi$.

Given that there are no restrictions on rank-1 logics other than the format of their axiomatisation, decidability is only automatic for finitely axiomatisable logics ([24, Theorem 3]. In particular, the additional assumption of decidability in Theorem 50 is not vacuously true. However, all rank-1 logics that appear in the literature are decidable, and we are not aware of a natural counterexample to decidability and present the following constructed rank-1 logic to demonstrate that the additional assumption of decidability in Theorem 50 is not vacuously true. (Embarrassingly, we are not aware of a recursively axiomatisable rank-1 logic that is undecidable.)

Example 51 (Not all rank-1 logics are decidable). Consider the similarity type $\Lambda=\mathbb{N}$ where each $n \in \mathbb{N}$ is nullary and let $U \subset \mathbb{N}$ be undecidable, but recursively enumerable. If $\mathcal{A}=$ $\{n \rightarrow \perp \mid n \in U\}$ then $L=\mathcal{L}(\mathcal{A})$ is undecidable.

As decidability for the logics introduced in Example 38 is known in the literature, interpolation is effective in all cases.

\section{CONCLUSION AND Discussion}

This paper answers two questions. The first question, often asked in the community, is whether one can formulate a version of Moss' coalgebraic logic [26] for which the endofunctor that defines the semantics does not need to preserve weak pullbacks. The second question, explicitly formulated in [36] is to what extent, and with what modifications, cover modalities can be used to generalise the interpolation result presented in op.cit.. Apart from answers to these questions, we have established several previously unknown interpolation results.

Conceptually, our answer to both questions mentioned above is the logic of exact covers. Concerning the first, we note that the logic of exact covers does not require the preservation of weak pullbacks. Regarding the second question, we have demonstrated that all rank-1 logics have the interpolation property via translating to and from the logic of exact covers. Apart from interpolation, we have given a complete tableau calculus and have proven the small model theorem and a Hennessy-Milner style result.

Our experience from dealing with the logic of exact covers indicates that it is conceptually much simpler than Moss' coalgebraic logic: for example, we have given a complete tableau calculus for the logic of exact covers whereas it has taken almost ten years for a complete axiomatisation of coalgebraic logic to appear [18]. A similar argument can be made for the distributive laws (see e.g. [21]) that underlie our treatment of bisimulation quantifiers. On the other hand, this simplicity comes at a price: the loss of monotonicity. However, 
logics based on the cover modality are very unlikely to be applicable to general neighbourhood frames [25], the simplest non-monotonic modal logic. Despite its conceptual simplicity, the logic of exact covers does have independent applications, as witnessed by the interpolation theorems of the previous section. In contexts where monotonicity is not required (such as e.g. interpolation) we would therefore consider the logic of exact covers ahead of Moss' coalgebraic logic as a tool to establish generic results.

The major question that this paper leaves open is to what extent the techniques presented here can be generalised to logics that are not rank-1. We have demonstrated in previous research that all non-iterative logics (i.e. logics that can be axiomatised by formulae with modal nesting depth $\leq 1$, such as e.g. the vast majority of conditional logics [28]) are complete with respect to the frame classes they define [30] and can be endowed with a cut-free sequent calculus [23], that is, they share important traits with rank-1 logics (for which the corresponding properties have been established in [39] and [31]). Both properties play a prominent role in interpolation proofs: interpolants via cut-elimination is a standard technique and is exercised e.g. in [5] for the prototypical non-iterative logic $T$, and completeness has been used in various places in the present paper. It is known that uniform interpolation does not hold for all modal logics (it fails e.g. for the iterative logic S4 [14]), but we are unaware of an example of a noniterative modal logic for which (uniform) interpolation fails. We therefore leave the following to future research:

Open Problem 52. Do all non-iterative modal logics (over finite similarity types) have (uniform) interpolation?

Acknowledgement. The research reported in this paper was conducted while visiting the Laboratoire dInformatique Fondamentale de Marseille (LIF) at the Université de Provence, France. We gratefully acknowledge fruitful and extended discussion with Lugi Santocanale during this visit, and the financial support provided by LIF that made this visit possible.

\section{REFERENCES}

[1] J. Adamek, V. Koubek, and V. Pohlova. Colimits in generalized algebraic categories. Acta Universitatis Carolinae, 13:311-324, 1972.

[2] J. Adámek and V. Trnková. Automata and Algebras in Categories. Kluwer, 1990.

[3] R. Alur, T. A. Henzinger, and O. Kupferman. Alternating-time temporal logic. J. ACM, 49(5):672-713, 2002.

[4] M. Bílková, A. Palmigiano, and Y. Venema. Proof systems for the coalgebraic cover modality. In C. Areces and R. Goldblatt, editors, Proc. AiML 2008, volume 7, pages 1-21, 2008.

[5] M. Bílková. Uniform interpolation and propositional quantifiers in modal logics. Studia Logica, 85(1):1-31, 2007.

[6] P. Blackburn, M. de Rijke, and Y. Venema. Modal Logic. Cambridge University Press, 2001.

[7] J. Burgess. Quick completeness proofs for some logics of conditionals. Notre Dame J. Formal Logic, 22:76-84, 1981.

[8] B. Chellas. Modal Logic. Cambridge, 1980.

[9] G. D'Agostino. Interpolation in non-classical logics. Synthese, 164(3):421-435, 2008.

[10] K. Fine. In so many possible worlds. Notre Dame J. Formal Logic, 13:516-520, 1972.

[11] T. French. Bisimulation Quantifiers for Modal Logic. PhD thesis, Department of Computer Science and Software Engineering, University of Western Australia, 2006
[12] S. Ghilardi. An algebraic theory of normal forms. Ann. Pure Appl. Logic, 71(3):189-245, 1995.

[13] S. Ghilardi. Unification in intuitionistic logic. J. Symb. Log., 64(2):859$880,1999$.

[14] S. Ghilardi and M. W. Zawadowski. Undefinability of propositional quantifiers in the modal system S4. Studia Logica, 55(2):259-271, 1995.

[15] H. H. Hansen, C. Kupke, and E. Pacuit. Neighbourhood structures: Bisimilarity and basic model theory. Logical Methods in Computer Science, 5(2), 2009.

[16] A. Heifetz and P. Mongin. Probabilistic logic for type spaces. Games and Economic Behavior, 35:31-53, 2001.

[17] S. Kraus, D. Lehmann, and M. Magidor. Nonmonotonic reasoning, preferentail models and cumulative logics. Artificial Intelligence, 14:167207, 1990.

[18] C. Kupke, A. Kurz, and Y. Venema. Completeness of the finitary Moss Logic. In C. Areces and R. Goldblatt, editors, Proceedings of AiML, volume 7, pages 193-217, 2008.

[19] C. Kupke and D. Pattinson. On modal logics of linear inequalities. In V. Goranko and V. Shehtman, editors, Proc. AiML 2010. College Publications, 2010.

[20] C. Kupke and D. Pattinson. Coalgebraic semantics of modal logics: an overview. Theoretical Computer Science, 412(38):5070-5094, 2011.

[21] C. Kupke and Y. Venema. Coalgebraic automata theory: Basic results. Logical Methods in Computer Science, 4(4), 2008.

[22] K. Larsen and A. Skou. Bisimulation through probabilistic testing. Inform. Comput., 94(1):1-28, 1991.

[23] B. Lellmann and D. Pattinson. Cut elimination for shallow modal logics. In K. Brünnler and G. Metcalfe, editors, Proc. TABLEAUX 2011, volume 6793 of Lecture Notes in Computer Science, pages 211-225. Springer, 2011.

[24] D. Lewis. Intensional logics without interative axioms. Journal of Philosophical Logic, 3:457-466, 1974.

[25] J. Marti and Y. Venema. Lax extensions of coalgebra functors. In D. Pattinson and L. Schröder, editors, Proc. CMCS 2012, volume 7399 of Lecture Notes in Computer Science, pages 150-169. Springer, 2012.

[26] L. Moss. Coalgebraic Logic. Ann. Pure Appl. Logic, 96:277-317, 1999.

[27] L. S. Moss. A note on expressive coalgebraic logics for finitary set functors. J. Log. Comput., 20(5):1101-1111, 2010.

[28] D. Nute and C. B. Cross. Conditional logic. In D. M. Gabbay and F. Guenthner, editors, Handbook of Philosophical Logic, volume 4, pages 1-98. Kluwer, 2001.

[29] E. Pacuit and S. Salame. Majority logic. In D. Dubois, C. A. Welty, and M.-A. Williams, editors, Proc. KR 2004, pages 598-605. AAAI Press, 2004.

[30] D. Pattinson and L. Schröder. Beyond rank 1: Algebraic semantics and finite models for coalgebraic logics. In R. Amadio, editor, Proc. FoSSaCS 2008, number 4962 in LNCS, pages 66-80, 2008.

[31] D. Pattinson and L. Schröder. Cut elimination in coalgebraic logics. Inf. Comput., 208:1447-1468, 2010.

[32] D. Pattinson. Coalgebraic modal logic: Soundness, completeness and decidability of local consequence. Theoret. Comput. Sci., 309:177-193, 2003.

[33] M. Pauly. A modal logic for coalitional power in games. J. Logic Comput., 12(1):149-166, 2002

[34] A. M. Pitts. On an interpretation of second order quantification in first order intuitionistic propositional logic. J. Symb. Log., 57(1):33-52, 1992.

[35] J. Rutten. Universal Coalgebra: A theory of systems. Theoret. Comput. Sci., 249(1):3-80, 2000.

[36] L. Santocanale and Y. Venema. Uniform interpolation for monotone modal logic. In L. D. Beklemishev, V. Goranko, and V. Shehtman, editors, Proc. AiML 2010, pages 350-370. College Publications, 2010.

[37] L. Schröder. Expressivity of coalgebraic modal logic: the limits and beyond. Theor. Comput. Sci., 390(2-3):230-247, 2008.

[38] L. Schröder and D. Pattinson. Rank-1 logics are coalgebraic. J. Logic Comput., 20(5):1113-1147, 2010.

[39] L. Schröder. A finite model construction for coalgebraic modal logic. J. Log. Algebr. Program., 73(1-2):97-110, 2007.

[40] Y. Venema. Automata and fixed point logics: a coalgebraic perspective. Inf. Comput., 204(4):637-678, 2006.

[41] Y. Venema. A modal distributive law (abstract). In D. Leivant and R. J. G. B. de Queiroz, editors, Proc. WoLLIC 2007, volume 4576 of Lecture Notes in Computer Science, page 351. Springer, 2007.

[42] A. Visser. Uniform interpolation and lauered bisimulation. In P. Hájek, editor, Gödel 96, pages 139-164. Springer, 1996. 